\title{
SPECTRUM OF A COMPOSITION OPERATOR
}

\author{
WILLIAM C. RIDGE
}

\begin{abstract}
A composition operator is a linear operator induced on a subspace of $K^{X}$ by a point transformation $\phi$ on a set $X$ (where $K$ denotes the scalar field) by the formula $T f(x)=f \circ \phi(x)$. Familiar examples include translation operators on the real line and on topological groups, analytic functions which preserve the class of harmonic functions (and Green's functions), ergodic transformations which induce unitary operators on $L^{2}$, shift and weighted shift operators.

The spectrum, approximate point spectrum, and point spectrum of an $L^{p}$-composition operator have circular symmetry about 0 , except on the unit circle, where they form unions of subgroups; certain consequences are derived from this.
\end{abstract}

Definition. If $X$ is a set, and $K$ the real or complex numbers, then $K^{X}$ is an algebra with operations defined pointwise on $X$. If $X_{1}$ is a subset of $X$, then a single-valued transformation $\phi: X_{1} \rightarrow X$ induces a homomorphism $T=T_{\phi}$ on $K^{X}$, defined for $f$ in $K^{X}$ by

$$
T f=(f \circ \phi) \chi_{x_{1}}
$$

where $\chi$ denotes the characteristic function. Such $T$ is called a composition operator; we say that $\phi$ induces $T$.

FAmiliar EXAmples. (1) Translation operators $T f(x)=f(x+a)$ on the real line; or $T f(t)=f(s t)$ on a topological group.

(2) The unitary operator on $L^{2}(X, \mu)$ induced by an ergodic transformation $\phi$ on $X: T f(x)=f \circ \phi(x)$.

(3) $X=X_{1}=$ complex plane, $\phi$ an analytic function; if $f$ is harmonic then so is $T f=f \circ \phi$.

(4) The left shift on $l_{+}^{2}$ (let $X=X_{1}=$ positive integers, $\phi(n)=n+1$ ); the right shift $\left(X_{1}=\right.$ positive integers except $\left.1, \phi(n)=n-1\right)$; and the bilateral shift on $l^{2}(X=$ integers, $\phi(n)=n-1)$.

(5) Weighted shifts: as in (4); assign suitable measures to the integers. [That is, represent as a shift on a weighted sequence space.]

(6) Any permutation (of an orthonormal basis) on a Hilbert space $H$ : let $\phi$ permute a corresponding set of atoms of $X, H=L^{2}(X)$.

Received by the editors January 24, 1972 and, in revised form, May 12, 1972. AMS (MOS) subject classifications (1970). Primary 28A65, 47A10, 47A35. 
(7) Any orthogonal projection on a Hilbert space: let $X=$ set of atoms representing the space, and $X_{1}=$ subset representing the range; $\phi=$ identity on $X_{1}$.

(8) Let $\left(e_{n}\right)_{n=-\infty}^{\infty}$ be an orthonormal basis of $l^{2}$, and let $S$ be the symmetry $S e_{n}=e_{-n}$. This has at least two natural representations as a composition operator:

(a) $X=$ integers with counting measure, $\phi(n)=-n, l^{2}=L^{2}(X)$;

(b) $X=(-\pi, \pi)$ with normalized Lebesgue measures, $\phi(x)=-x$.

$L^{2}(X)$ has an orthonormal basis $t_{n}(x)=e^{i n x}$, and

$$
T_{\phi} t_{n}(x)=t_{n} \circ \phi(x)=t_{n}(-x)=e^{-i n x}=t_{-n}(x),
$$

so $T_{\phi}$ is unitarily equivalent to $S$.

ElEMENTARY FACTS. These are routine to check, and may be found in [1] or [2].

$T_{\phi \cdot \psi}=T_{\psi} \circ T_{\phi}$. If $\phi$ is invertible, then so is $T_{\phi}$, and $\left(T_{\phi}\right)^{-1}=T_{\phi^{-1}}$. If $E \subset X$, then

$$
T \chi_{E}=\left(\chi_{E} \circ \phi\right) \chi_{x_{1}}=\chi_{\phi^{-1} E} \text {. }
$$

Suppose $(X, \mu)$ is sigma-finite and $\phi$ is measurable. We define another measure on $X$ :

$$
\mu_{\phi}(E)=\mu\left[\phi^{-1}(E)\right] .
$$

Let $N$ denote the functions vanishing $\mu$-almost everywhere. Then $T N \subset N$ if and only if $\mu_{\phi} \ll \mu$, then the Radon-Nikodym derivative $d \mu_{\phi} / d \mu$ exists, also $T$ induces a natural homomorphism on $K^{X} / N$ (which we shall also call $T$ ).

Then $T L^{\infty}(X) \subset L^{\infty}(X),\|T\| \leqq 1$, and $T$ is an $\left(L^{\infty}-\right)$ isometry if and only if $\mu$ is also absolutely continuous with respect to $\mu_{\phi}$.

$T L^{p}(X) \subset L^{p}(X), 1 \leqq p<\infty$, if and only if $d \mu_{\phi} / d \mu$ is essentially bounded on $X$. In this case,

$$
\|T\|=\left\|\frac{d \mu_{\phi}}{d \mu}\right\|_{\infty}^{1 / p}
$$

The lower bound of $T, \inf (\|T f\|:\|f\|=1)$, equals ess $\inf \left(d \mu_{\phi} / d \mu\right)$.

The nullspace $N(T)$ is $L^{p}\left(X_{0}\right)$, where $X_{0}=\left\{x:\left(d \mu_{\phi} / d \mu\right)(x)=0\right\}$, and the range $R(T)$ is $L^{p}\left(X_{1}\right)$.

SPECTRUM. We assume that $X$ is sigma-finite, and $\phi$ induces an operator $T$ on $L^{p}(X)$. We examine the spectrum of such an operator, and hence of any operator which can be so represented.

Let $K$ denote the complex numbers, $C$ the complex unit circle, $\Lambda(T)$ the spectrum of $T, \Pi$ the approximate point spectrum (the set of complex numbers $c$ such that $T-c$ is not bounded below), $\Pi_{0}$ the point spectrum, 
and $\Gamma$ the compression spectrum (the set of complex numbers $c$ such that $T-c$ does not have dense range).

ELEMENTARY Facts. 0 is in $\Pi$ if and only if ess $\inf \left(d \mu_{\phi} / d \mu\right)=0.0$ is in $\Pi_{0}$ if and only if this essential infimum is attained on a set of positive measure.

If $X$ is nonatomic, then the nullity of $T$ is either zero or infinite. For if $d \mu_{\phi} / d \mu$ vanishes on a set $E$ of positive measure, then $T=0$ on $L^{p}(E)$ which has infinite dimension.

Consequence. The left shift has no nonatomic representation as a composition operator.

THEOREM A. $\Pi \cap C$ is a union of subgroups of $C$.

Proof. Suppose $e^{i a}$ is in $\Pi$, a real; for any positive integer $n$, and $\varepsilon>0$, choose $f$ of unit norm such that $\left\|T f-e^{i a} f\right\|<\varepsilon / n$. Define $g$ on $X$ by

$$
g(x)=|f(x)| \exp [\operatorname{in} \arg f(x)]
$$

on spt $f$ (the set of $X$ such that $f(x) \neq 0$ ), and $g=0$ elsewhere. Then $\|g\|=\|f\|=1$.

Let $D=\{x: f(x)=0$ or $T f(x)=0\}$. If $x$ is in $D$, then

$$
\left|T g(x)-e^{i n a} g(x)\right|=\left|T f(x)-e^{i a} f(x)\right|,
$$

since one of the terms on each side vanishes, and the others by definition have the same modulus.

We pause for two propositions.

(1) If $s \geqq 1$ then $\left|1-e^{i t}\right| \leqq\left|1-s e^{i t}\right|$ for all real $t$.

Proof. Geometrically, draw the radius from 0 to $s e^{i t}$ (through $e^{i t}$ ); the greater side subtends the greater angle in the triangle $\left(1, e^{i t}, s e^{i t}\right)$. Analytically, let $h(x)=\left|1-x e^{i t}\right|^{2}$ for real $x$. Then $h^{\prime}(x)=1+2(x-\cos t)>0$ if $x \geqq 1$. So $h(s) \geqq h(1)$.

(2) If $r, s$ are positive, $b, c$ are real, and $n$ is a positive integer, then $\left|r e^{i n b}-s e^{i n c}\right| \leqq n\left|r e^{i b}-s e^{i c}\right|$.

Proof. If $s \geqq 1$, then, by (1),

$$
\begin{aligned}
\left|1-s e^{i n t}\right| & \leqq\left|1-e^{i t}\right|+\left|e^{i t}-e^{2 i t}\right|+\cdots+\left|e^{(n-1) i t}-s e^{i n t}\right| \\
& \leqq n\left|1-s e^{i t}\right|
\end{aligned}
$$

which is equivalent to (2).

Now if $x$ is not in $D$, then, using (2),

$$
\begin{aligned}
\left|T g(x)-e^{i n a} g(x)\right| & \\
& =|| T f(x)|\exp \{\operatorname{in} \arg T f(x)\}-| f(x)|\exp \{\operatorname{in}[a+\arg f(x)]\}| \\
& \leqq n|| T f(x)|\exp \{i \arg T f(x)\}-| f(x)|\exp \{i[a+\arg f(x)]\}| \\
& =n\left|T f(x)-e^{i a} f(x)\right| .
\end{aligned}
$$


Integrating over $D$ and $X-D$, we have $\left\|T g-e^{i n a} g\right\| \leqq n\left\|T f-e^{i a} f\right\|$, which is less than $\varepsilon$. So $e^{i n a}$ is in $\Pi$.

If $e^{i a}$ is a complex root of unity, contained in $\Pi$, then, by the above argument, $\Pi$ contains the entire subgroup of $C$ generated by $e^{i a}$. Otherwise, the multiples of $e^{i a}$ are dense in $C$, and since $\Pi$ is closed, it contains C.

COROllaRy. $\Pi_{0} \cap C$ is a union of subgroups of $C$.

Proof. Set $\varepsilon=0$ in the above proof.

Note. Every union of subgroups of $C$ is the point spectrum of some composition operator. Suppose $G$ is the subgroup of order $n$ generated by a primitive $n$th root $w$ of unity. Let $X$ be a set of $n$ atoms, $x_{1} \cdots x_{n}$; let $\phi\left(x_{k}\right)=x_{k+1}, k=1, \cdots, n-1 ; \phi\left(x_{n}\right)=x_{1} ;$ let $f\left(x_{k}\right)=w^{-r k}$. Then $T_{\phi} f=w^{r} f$, and $\Pi_{0}(T)=G$.

If $G$ is a union of such groups $G_{a}$, let $X$ be the disjoint union of $\phi$-cycles $X_{a}$ as above; $L^{2}(X)$ is then the orthogonal sum of the $L^{2}\left(X_{a}\right)$, and $\pi_{0}(T)=$ $G$. Finally, if $G$ is infinite cyclic, let $X$ be an infinite $\phi$-cycle.

THEOREM B. $\Pi-C$ has circular symmetry about 0 .

Proof. Suppose $c$ is in $\Pi,|c| \neq 0,1$, and $\|T f-c f\|<\varepsilon,\|f\|=1$. Then $\|T|f|-|c f|\|<\varepsilon$. Thus $|c|$ is in $\Pi$, and we may assume $c>0, f \geqq 0$.

Suppose $a=c e^{i t}, 0 \leqq t<2 \pi$. Define $g$ on $X$ by

$$
g(x)=f(x)\left\{\exp \text { it } \log _{c} f(x)\right\}
$$

on spt $f$, and $g=0$ elsewhere. Then $\|g\|=\|f\|=1$, and $|T g(x)| \equiv|T f(x)|$.

Let $D=\{x: T f(x)$ or $f(x)=0\}$. If $x$ is in $D$, then $|T g(x)-a g(x)|=$ $|T f(x)-c f(x)|$.

If $x$ is not in $D$, let $q(x)=\operatorname{minimum}\{T f(x), c f(x)\}$. By the triangle inequality

$$
\begin{aligned}
|T g(x)-\operatorname{ag}(x)| \leqq & || T g(x)|-| \operatorname{ag}(x) \| \\
& +q(x)|\exp [i \arg T g(x)]-\exp [i \arg a g(x)]| .
\end{aligned}
$$

The first summand is equal to $|T f(x)-c f(x)|$. Since

$$
\arg a g(x)=\arg a+t \log _{c} f(x)=t\left(1+\log _{c} f(x)\right)=t \log _{c} c f(x)
$$

we also have

$$
\begin{aligned}
|\exp [i \arg T g(x)]-\exp [i \arg a g(x)]| & \leqq|\arg T g(x)-\arg \operatorname{ag}(x)| \\
& =t\left|\log _{c} T f(x)-\log _{c} c f(x)\right| .
\end{aligned}
$$

Since $|d| d s\left(\log _{c} s\right) \mid=(s \log c)^{-1} \leqq(q(x) \log c)^{-1}$ for $s \geqq q(x)$, we have $\left|\log _{c} T f(x)-\log _{c} c f(x)\right| \leqq(q(x) \log c)^{-1}|T f(x)-c f(x)|$. 
Consequently

$$
\begin{aligned}
|T g(x)-a g(x)| & \leqq|T f(x)-c f(x)|(1+t /(\log c)) \\
& \leqq|T f(x)-c f(x)|(1+2 \pi /(\log c)) .
\end{aligned}
$$

Integrating over $D$ and $X-D$, we have

$$
\|T g-a g\| \leqq(1+2 \pi /(\log c)) \varepsilon
$$

and so $a$ is in $\Pi$.

CoROllaRy 1. $\Pi_{0}-C$ has circular symmetry about 0 .

Proof. Set $\varepsilon=0$ above.

COROLlary 2. The residual spectrum $\Gamma-\Pi$, minus $C$ (and hence $\Lambda-C$ ) has circular symmetry about 0 .

Proof. If $a$ is in $\Gamma-\Pi$ and $b$ is not, with $|a|=|b| \neq 0,1$, then $b$ is not in $\Pi$ (otherwise $b$, and hence $a$, would be in $\Pi$ ). $\Gamma$ then must have a boundary point $c$ on the same circle; necessarily $c$, and hence $a$, is in $\Pi$, a contradiction.

COROLlaRY 3. If $T$ is a compact composition operator, then $\Lambda-\{0\}$ is a finite subset of $C$. (Hence, $T=A+N$ where $A$ has finite rank and $N$ is quasinilpotent.)

Proof. $T$ cannot have a whole circle of spectral values bounded away from 0 .

COROLlARY 4. Every selfadjoint composition operator on $L^{2}$ is a partial symmetry; every positive composition operator is a projection.

Proof. If $T$ is selfadjoint then $\Lambda$ is real, hence contained in $\{-1,0,1\}$; if $T$ is positive then $\Lambda \subset\{0,1\}$.

THEOREM C. If $X$ has finite measure, then $\Pi_{0}-\{0\}$ is either connected or contained in $C$.

Proof. Suppose $T f=c f, c>0$. Then we may assume $f \geqq 0$. For any positive integer $n$, taking positive roots, $f^{1 / n}$ is in $L^{2}$ since it is dominated by $1 \vee f$, and $T\left(f^{1 / n}\right)=(T f)^{1 / n}=c^{1 / n} f^{1 / n}$.

If $m \leqq n$, then $f^{m / n}$ is also in $L^{2}$ and $T f^{m / n}=c^{m / n} f^{m / n}$.

If $0 \leqq a \leqq 1$ and a rational sequence $r_{k}$, in the unit interval, converges to $a$, then $f^{r_{k}}$ converges to $f^{a}$ pointwise, hence in $L^{2}$ (dominated convergence). 
So

$$
T\left(f^{a}\right)=\lim T\left(f^{r_{k}}\right)=\lim c^{r_{k}} f^{r_{k}}=c^{a} f^{a}
$$

and $c^{a}$ is in $\Pi_{0}$. Thus $\Pi_{0}$ contains the closed interval between 1 and $c$.

Now if $\Pi_{0}-\{0\}$ contains any point outside $C$, then by Theorem $B$ (Corollary 1) and the preceding paragraph, it comprises a whole annulus, possibly excluding part of $C$ but necessarily including 1 , and hence is connected.

EXAMPLES. Composition operators and their spectra.

(1) $X=C, \phi(z)=w z, w$ a primitive $n$th root of 1 . If $T f=c f$ then $c^{n} f(z)=$ $T^{n} f(z)=f\left(w^{n} z\right)=f(z)$ so $c^{n}=1$. So $\Pi_{0}$ is a finite subgroup of $C$. If $A$ is a nonnull subset of $\operatorname{arc}(1, w)$, let $f=\sum_{k} w^{k s} \chi_{\phi^{k} A}$. We easily find $T f=w^{s} f$. So all eigenvalues of $T$ have infinite multiplicity.

(2) $X=(0,1)$, Lebesgue measure, $I_{0}=\left(\frac{1}{3}, \frac{2}{3}\right), I_{1}=\left(\frac{2}{3}, \frac{5}{6}\right), I_{2}=\left(\frac{5}{6}, \frac{11}{12}\right), \cdots$, $I_{-1}=\left(\frac{1}{6}, \frac{1}{3}\right), I_{-2}=\left(\frac{1}{1}, \frac{1}{6}\right), \cdots ; \phi$ maps $I_{k}$ linearly onto $I_{k+1}$.

If $A_{0} \subset I_{0}$ and $A_{k}=\phi^{k} A_{0}$, let $f=\sum c^{k} \chi_{A_{k}}$. Then

$$
\|f\|^{2}=\mu\left(A_{0}\right)\left(\sum_{m=0}^{\infty} 2^{-m}|c|^{2 m}+\sum_{m=1}^{\infty} 2^{-m}|c|^{-2 m}\right),
$$

and both series converge if and only if $1 / \sqrt{ } 2<|c|<\sqrt{ } 2$. Then $T f=c f$, and $\Pi_{0}$ is precisely this open annulus. $\Pi$ is the closed annulus, because of the upper and lower bounds on $d \mu_{\phi} / d \mu$.

Obviously the radii of this annulus can be changed by changing the relative lengths of the $I_{k}$. If we allow infinite measure, then the annulus can have any two positive radii. ( $X=\bigcup I_{k}$ could be the whole real line, and lengths of successive $I_{k}$ could have any ratio.)

By considering the disjoint union of two such spaces with disjoint annuli as the corresponding spectra, we see that Theorem $\mathrm{C}$ does not hold if we allow infinite measure.

(3) $X=$ positive integers with $\mu(n)=a^{2 n}, 0<a<1, \phi(n)=n-1, n=2$, $3, \cdots$. Note that $\mu(X)$ is finite. $T$ is a weighted shift; $\Pi$ is the circle of radius $a$ about $0 ; \Lambda$ is the entire disk of the same radius. (More properly, $T$ is a shift on a weighted sequence space.)

We now see that Theorem $C$ does not hold with $\Pi$ in place of $\Pi_{0}$.

(4) $X=$ real line, $\mu(A)=\int_{A} e^{x} d x, \phi(x)=x-1$. Here

$$
\mu_{\phi}(A)=\int_{\phi^{-1} A} e^{x} d x=\int_{A} e^{x+1} d x=e \mu(A),
$$

so $d \mu_{\phi} / d \mu \equiv e$ and $\Pi$ is the circle about 0 of radius $\sqrt{ } e$.

Since $T$ has dense range (including $\chi_{A}, A$ bounded), 0 is not in $\Gamma$ and hence not in the spectrum. 
Nordgren [3] has worked out some special cases when $X$ is the unit circle with normalized Lebesgue measure.

\section{REFERENCES}

1. N. Dunford and J. T. Schwartz, Linear operators. I: General theory, Pure and Appl. Math., vol. 7, Interscience, New York, 1958. MR 22 \#8302.

2. W. C. Ridge, Composition operators, Thesis, Indiana University, Bloomington, Ind., 1969, pp. 1-46.

3. E. Nordgren, Composition operators, Canad. J. Math. 20 (1968), 442-449. MR 36 \#6961.

Department of Mathematics, Indiana and Purdue Universities at Indianapolis, INDIANAPOLIS, INDIANA 46205 